\title{
Synovial tuberculosis of the hand: An ancient disease in an unusual localisation
}

\author{
Renato Franz Matta Ramos, Lucas Cancian'1, Fernando Calcagnotto ${ }^{1}$, Ricardo Zeni', \\ Gabriel Varela1, Tamsin Burgues², Jefferson Braga Silva1 \\ Plastic Surgery Department, Bliss Plastic Surgery Clinic, Lima, Peru, ${ }^{1}$ Hand and Reconstructive Microsurgery Department and \\ School of Medicine, Saint Lucas Hospital, Pontifical Catholic University of Rio Grande do Sul, Porto Alegre, ${ }^{2}$ General Surgery \\ Department, Santa Casa da Misericordia Hospital, Rio de Janeiro, Brazil.
}

Address for correspondence: Dr. Renato Franz Matta Ramos, Sanchez Carrion Avenue, 417 of 402, San Isidro, Lima 41, Peru. E-mail: renatomatta82@hotmail.com

\section{ABSTRACT}

Background: Tuberculosis is the most prevalent infectious disease in the world. It is mainly caused by Mycobacterium tuberculosis. Osteoarticular tuberculosis represents $1 \%-3 \%$. Tenosynovitis is the most common form of the disease in the hand. Aims: The aim of this study is to present an update of synovial tuberculosis. Materials and Methods: The authors present a literature review, the clinical and surgical management and case reports. Results: The outcomes were satisfactory and were not report complications. Conclusions: Early diagnosis, surgical transection of the transverse carpal ligament, debridement and complete excision of the infected synovium may be required, along with antituberculosis drugs. Knowledge of this disease in the hand can provide a better diagnosis and outcome.

\section{KEY WORDS}

Hand; joint; synovial; tuberculosis

\section{INTRODUCTION}

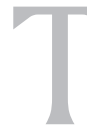

uberculosis is the most prevalent infectious disease in the world. It is mainly caused by Mycobacterium tuberculosis. Latent infection occurs in about 2 billion people. Approximately 8 million people/year develop the active form. ${ }^{[1,2]}$ Tuberculosis in the hand is manifested as osteomyelitis in small bones (carpal bones), metacarpals and phalanges. ${ }^{[1]}$

\begin{tabular}{|l|l|}
\hline \multicolumn{2}{|c|}{ Access this article online } \\
\hline Quick Response Code: & Website: \\
\hline & www.ijps.org \\
\hline
\end{tabular}

\section{EPIDEMIOLOGY}

The Southeast Asia accounts for $40 \%$ of cases worldwide..$^{3]}$ In the US, $10 \%$ of extrapulmonary tuberculosis (EP-TBC) cases occur in HIV-infected patients, and in Africa, one-third of adults with osteoarticular TBC (O-TBC) are HIV-positive. ${ }^{[4]}$ In children, $20 \%$ of all mycobacterial infections are EP-TBC. ${ }^{[4,5]}$ The most common EP-TBC sites

This is an open access article distributed under the terms of the Creative Commons Attribution-NonCommercial-ShareAlike 3.0 License, which allows others to remix, tweak, and build upon the work non-commercially, as long as the author is credited and the new creations are licensed under the identical terms.

For reprints contact: reprints@medknow.com

How to cite this article: Matta Ramos RF, Cancian L, Calcagnotto F, Zeni R, Varela G, Burgues T, et al. Synovial tuberculosis of the hand: An ancient disease in an unusual localisation. Indian J Plast Surg 2017;50:130-7. 
are lymph nodes, genitourinary tract, bone marrow, central nervous system (CNS) and musculoskeletal system, which include bones, joint, bursas and tendons or tenosynovium. ${ }^{[6]}$

O-TBC represents $1 \%-3 \%$ of all cases and $5 \%-10 \%$ of EP-TBC, followed by pleural and lymphatic tuberculosis. It is usually monoarticular and located in joints that support body weight, such as the vertebrae, knee and hip; $;^{[1-3,7-17]}$ some locations such as the scapula are rare. ${ }^{[3]}$ In developed countries, O-TBC usually affects people over 55 years of age; while in immigrants, it is more common in individuals of 20-35-years old. Concomitant pulmonary involvement is diagnosed in up to $29 \% \cdot{ }^{[4]}$ Tenosynovitis is the most common form of the disease in the hand. ${ }^{[18-20]}$ It is important to note that individuals with advanced HIV infection are more likely to have EP-TBC such as musculoskeletal manifestations. ${ }^{[21]}$

Risk factors are the local conditions of joints and bones (trauma), immunosuppressed patients (HIV), immunosuppressive drug users (corticosteroids), patients with rheumatoid arthritis and systemic lupus erythematosus (SLE), smoking, alcoholism and patients with joint prostheses. ${ }^{[1-3,7-13]}$

\section{PATHOPHYSIOLOGY}

Primary tuberculosis infection occurs by inhalation where the main target is the lung. ${ }^{[1,2,22]}$ Transmission of M. tuberculosis occurs only by air into a ventilated lung area. Musculoskeletal tuberculosis occurs, in most cases, through haematogenous dissemination from the primary focus. In immunosuppression circumstances, it is reactivated. Lymphatics are other pathways to spread it. ${ }^{[5]}$

Innate and acquired immunity are important for disease spread. Macrophages phagocyte pathogens, release cytokines and cause inflammation. Bacteria that survive phagocytosis grow into the cells, destroy them and spread through the blood to distant organs. ${ }^{[1,2,7]}$ The pathogens are transported by macrophages to the lymph nodes, and here, they activate acquired immunity mediated by CD4+ lymphocytes. The result of this inflammatory process is a granuloma. The microorganisms remain in epithelial cells surrounded by lymphocyte rings forming a structure called a tuberculoma, with necrosis in the central region, forming an abscess. Afterwards, the microorganisms are remained inactive in different regions of the body for life. Instability within the immune system affects the disease control and secondary tuberculosis can develop, which can lead to the reactivation of primary tuberculosis. ${ }^{[1,2,22]}$

Apparently, M. tuberculosis is able to persist in adipose tissue, suggesting that this may be a source of EP-TBC. ${ }^{[23]}$

Joint infection is derived from adjacent osteomyelitis or by contact of synovial fluid with blood, where the cartilage undergoes necrosis. Vascularised bones such as the vertebrae are targets of microorganisms. ${ }^{[1-3,7-10,13]}$

Infrequently, the bacillus (Koch's bacillus) is spread to the spine along the paravertebral venous plexus of Batson or by lymphatic drainage to paraaortic lymph nodes. ${ }^{[4]}$

\section{HAND TUBERCULOSIS}

Tuberculosis in the hand is manifested as osteomyelitis in small bones (carpal bones), metacarpals and phalanges ${ }^{[1]}$ or as dactylitis. ${ }^{[12]}$ There is phlogosis in the bone, and constitutional symptoms can occur, such as fever, night sweats, weight loss and anorexia. ${ }^{[1,22]}$

Tenosynovitis is the most common form. It presents gradually and for unknown reasons, the flexor side is more affected than the extensor, as well as the ulnar side compared to the radial one. Tenosynovitis is also more frequent in the arms when compared to the lower limbs, and it often is found of the dominant arm. ${ }^{[24]}$ The delay in diagnosis may result in tendon rupture. Furthermore, muscles and nerves are resistant to infection. ${ }^{[22]}$ Compound palmar ganglion also known as chronic flexor tenosynovitis is a rare manifestation of the flexor tendons at the wrist or palm and is the tubercular infection affecting the tenosynovium of long flexor tendons. ${ }^{[6]}$ There are many differential diagnoses that should also be thought about when considering compound palmar ganglion. These include gouty arthritis, rheumatoid arthritis, sarcoidosis, pyogenic infection, a possibly infected ganglion, foreign body tenosynovitis and fungal infection, pigmented villonodular synovitis of tendon sheath, amyloidosis and synovial chondromatosis. ${ }^{\mid 25]}$

Occasionally, the mass can compress the median nerve

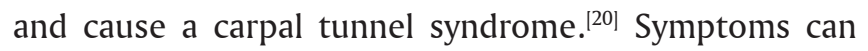
include pain, finger paraesthesia, hand edema, thenar atrophy, Phalen and Tinel signs, elevated red blood cell 
sedimentation rate and alteration in electrodiagnostic

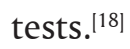

In general, tenosynovitis evolves slowly with pain and functional limitation and manifests as 'a sausage shape mass' along the inflamed tendons. There are other rare forms of tuberculosis in the hand, such as cutaneous tuberculosis or bursitis. ${ }^{[22]}$ Marquet et al. described a case of verrucous tuberculosis caused by Mycobacterium bovis causing carpal joint infection on the contralateral side. ${ }^{[26]}$

Synovial biopsy is important for diagnosis. Imagine generally assists in the diagnosis and can reveal a decrease in ossification and in the diameter of the synovial sheath. Diagnosis is difficult when presented as polyarticular, causing similar symptoms such as rheumatoid arthritis. ${ }^{[18-20]} \mathrm{X}$-rays of the hand bones can reveal cysts, lytic images and joint destruction. Magnetic resonance imaging (MRI) is not specific but helps to determine the extent of the lesion. ${ }^{[22]}$

The synovial sheath is replaced by 'rice bodies', which are fibrinous masses present in $50 \%$ of cases of tuberculosis. ${ }^{[18-20]}$

Early diagnosis, surgical transection of the transverse carpal ligament, debridement and complete excision of the infected synovium may be required, accompanied by treatment with antituberculosis drugs. Post-operative drug combinations are used for 1 year (rifampicin, ethambutol, isoniazid and pyrazinamide). ${ }^{[18]}$ Other procedures for joint lesions can be considered when there is severe cartilage destruction and poor response to drug therapy. ${ }^{[19,20]}$

\section{DIAGNOSIS}

Diagnosis can be difficult, and delay can cause harm, but most people with EP-TBC can be cured if they have access to diagnosis and treatment with anti-TB drugs in time. For this reason, when facilities permit, all patients suspected of having EP-TBC should have appropriate samples taken for microbiological and/or histological testing. As there is an extensive list of differential diagnoses for tubercular tenosynovitis plus the fact that non-specific clinical, radiological and histopathological findings can significantly delay both the diagnosis and management leading to complications. The best method to make a concluding diagnosis is open biopsy and mycobacterial culture; especially in acute suppurative tenosynovitis, where synovial sheath fluid is the predominant feature. Laboratory findings are generally negative, except for the erythrocyte sedimentation rate, witch is usually increased. However, the culture usually needs some weeks to reach a diagnosis. The positive result of synovial fluid acid-fast bacteria staining, culture of mycobacterium, histology and polymerase chain reaction (PCR) are $32 \%$, $80 \%, 65 \%$ and $63 \%$, respectively, and PCR can be used at the beginning for initial diagnosis. ${ }^{\mid 6,27,28]}$

The presence of alcohol-acid-resistant bacilli (AARB) on Ziehl-Neelsen staining may indicate tuberculosis, but this is not pathognomonic (positive in only 20\%). Definitive diagnosis is by isolation of Koch's bacillus in synovial fluid. Culture has more specificity and a positivity of $80 \%,{ }^{[5]}$ so biopsy confirms the diagnosis. Analysis of protein and glucose levels is non-specific. ${ }^{[1,2,7-14]}$ Histopathological examination shows caseous necrosis, epithelial granuloma and Langhans type giant cells.

Microbial culture is positive in $80 \%$ of cases,,$^{[1,8]}$ but sterile cultures can result in partially treated disease. ${ }^{[7]}$ In endemic areas, if the patient exhibits clinical and radiological aspects of the disease, the administration of preventive antimicrobial drugs can be started without the need of microbiological culture. ${ }^{[22]}$

The purified protein derivative test (PPD) may indicate the presence of $M$. tuberculosis (but false negatives are frequent). ${ }^{[1-3,9,13,14,22]}$

A simple X-ray may not be useful in the early stages of the disease or may be non-specific. Pelayo et al. published a protocol for the diagnosis and treatment of septic arthritis. They recommended performing imaging tests before the histological and microbiological tests to eliminate others diagnostic hypotheses. In tuberculous arthritis, the Phemister triad can be observed (articular osteoporosis, bone erosion and reduced joint space). Ultrasound can guide joint drainage..$^{[1,7-10,13,17]}$

\section{TREATMENT}

The prevention of the disease is possible maintaining personal hygiene and good nutrition. Effectivetuberculosis treatment depends on early diagnosis and knowledge of its extent and complications. Pharmacological treatment requires more than one drug to prevent drug resistance. 
Prolonged treatment is necessary to reach the difficult to access bacilli locations, avoiding a new manifestation in the future. The most commonly used antibiotics against tuberculosis are isoniazid, rifampicin, pyrazinamide and ethambutol, where isoniazid and rifampicin are the most effective. ${ }^{[1-3,7,9,12,13,22]}$

The WHO recommends the inclusion of pyrazinamide and ethambutol (or streptomycin) in the first 2 months. O-TBC in children requires at least 12 months of treatment. Personal monitoring is critical for a successful outcome. Erythrocyte sedimentation rate and serum protein $\mathrm{C}$ values are useful for monitoring the patient's treatment. . $^{[1,3,7,9,10]}$

Occasionally, surgery is recommended with specific guidelines when treatment does not entirely work and one of the following complications occurs: Nerve compression, impending bone collapse, joint debridement, drainage of large abscesses and correction of deformity in healed disease, where debridement, abscess drainage, removal of ovoid bodies and synovectomy, are required. ${ }^{[1,7,9-13,22,27]}$ When the tendon repair is performed, the immobilisation is recommended for 14 days and early physiotherapy is indicated to avoid tendon adherence and tenodesis.

\section{MATERIALS AND METHODS}

The authors present a case series of synovial tuberculosis on the hand and the management performed in the hand and microsurgery department.

\section{CASE REPORTS}

\section{Case 1}

A 61-year-old male patient with asymmetric and progressive increased tumours on the right wrist with 3 years of progression. During the examination, it was demonstrated flexion and extension finger's limitation. The patient had a family history of pulmonary tuberculosis [Figure 1]. The X-ray of the right wrist shows radiolucid lesions in carpal bones, reduction of intercarpal joint spaces and swelling of the soft tissues [Figure 2]. A cavity was observed after a complete synovectomy [Figure 3].

\section{Case 2}

A 47-year-old male patient with a tumour on the volar and ulnar side of the wrist with 9 months of progression. The clinical examination presented

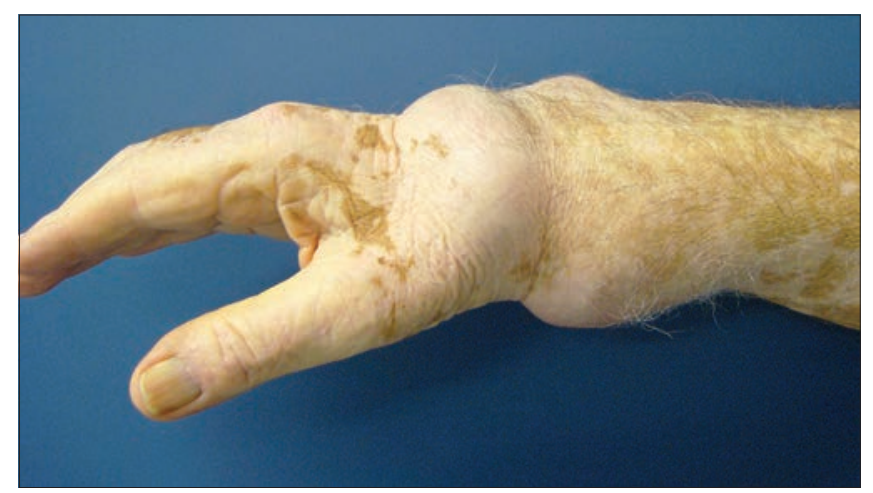

Figure 1: Right hand tumour

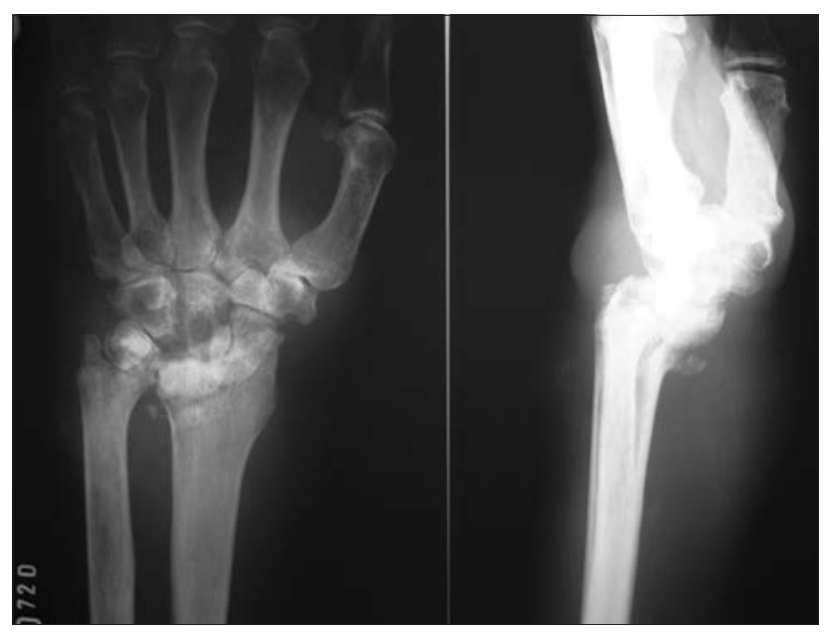

Figure 2: X-ray details

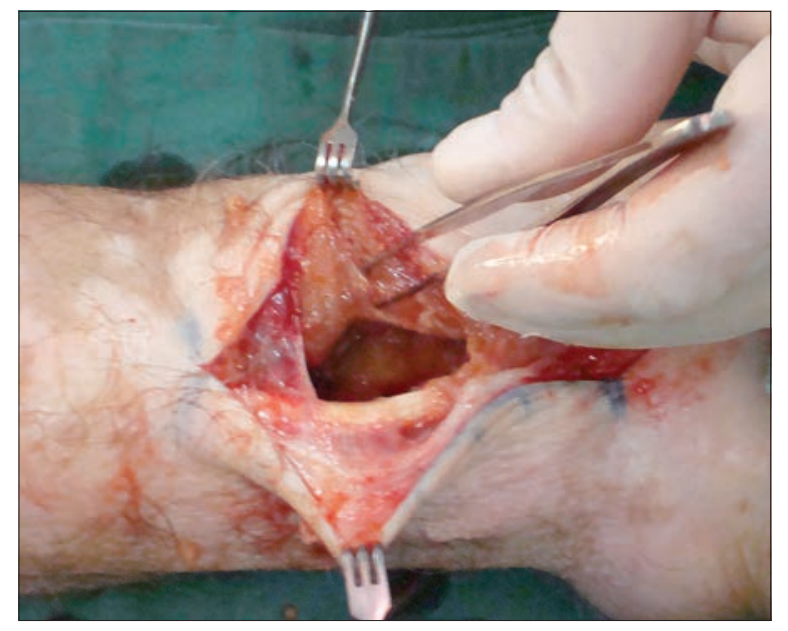

Figure 3: Cavity after synovectomy

fingers and wrist flexion limitation. He was diagnosed of synovial cyst and treated twice with direct puncture without improvement. There was no family history of pulmonary tuberculosis [Figure 4]. During surgery, it was observed an extensive hypertrophy of the synovial capsule of the flexor tendons and 'rice bodies' around the tendon [Figure 5].

Indian Journal of Plastic Surgery Volume 50 Issue 2 May-August 2017 


\section{Case 3}

A 65-year-old male patient with a tumour on the index finger associated with pain with 3 months of progression. The right-hand radiography shows soft-tissue augmentation of the volar side of the right index finger, without osteoarticular changes [Figure 6]. The right hand MRI shows synovial tissue hypertrophy corresponding to the flexor tendons of the index finger [Figure 7].

\section{Case 4}

A 38-year-old male patient with a tumour on the volar and ulnar side of the left wrist with 5 months of progression. The clinical examination presented intense pain during flexion-extension of the fourth and fifth fingers. The patient has a brotherwith pulmonary tuberculosis [Figure 8]. During surgery, synovial capsule hypertrophy with 'rice bodies' structures was observed [Figure 9]. Computed tomography of the left wrist shows peritendinous tissue hypertrophy and compression of adjacent structures [Figure 10].

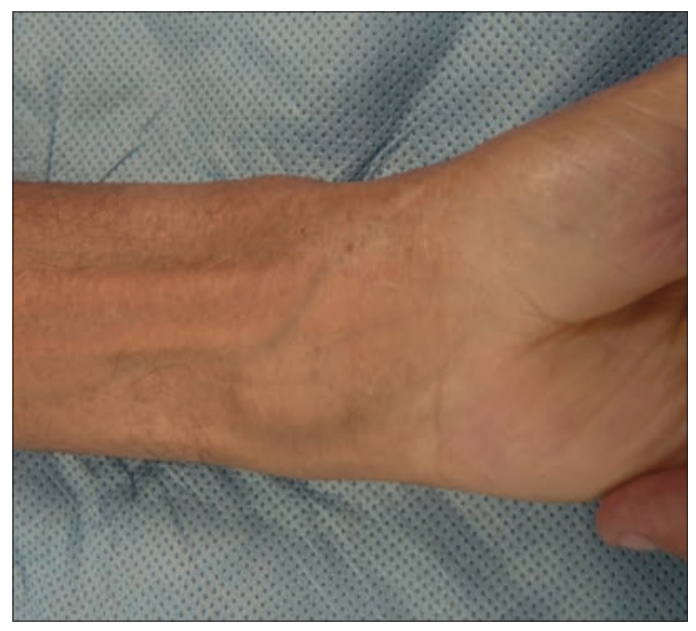

Figure 4: Wrist tumour

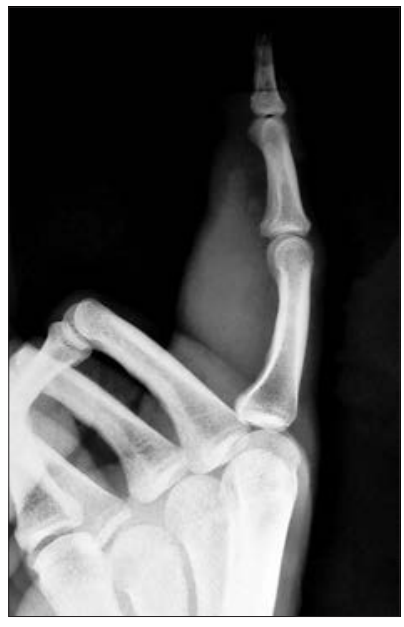

Figure 6: X-ray of index finger

\section{Case 5}

A 39-year-old male patient with a tumour on the volar and ulnar side of the right wrist, associated with pain and flexion limitation of the fingers and with 2 years of progression. He reported swelling in the thumb and fifth finger in the last 4 months [Figure 11]. During surgery, an extensive hypertrophy of synovial tissue and 'rice bodies' structures, were observed [Figure 12].

\section{RESULTS}

All patients evolved satisfactory with the initial surgical management and 1-year pharmacological treatment. The authors recommended an early physiotherapy of all patients, usually after the $7^{\text {th }}$ post-operative days.

Case 2 result at 12-month post-operatively. Synovectomy with excision of hypertrophied tissues, combined with antituberculosis chemotherapy [Figure 13].

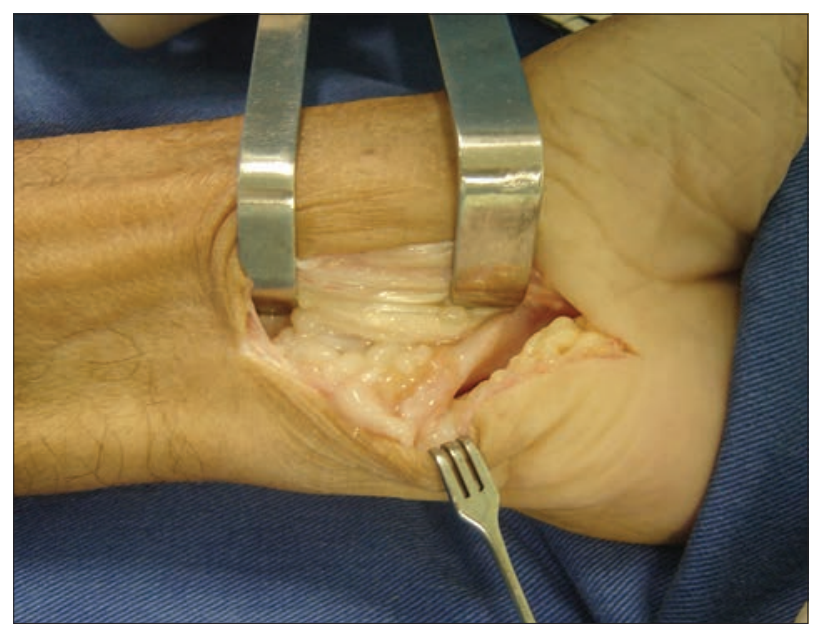

Figure 5: Extensive synovial hypertrophy and rice bodies

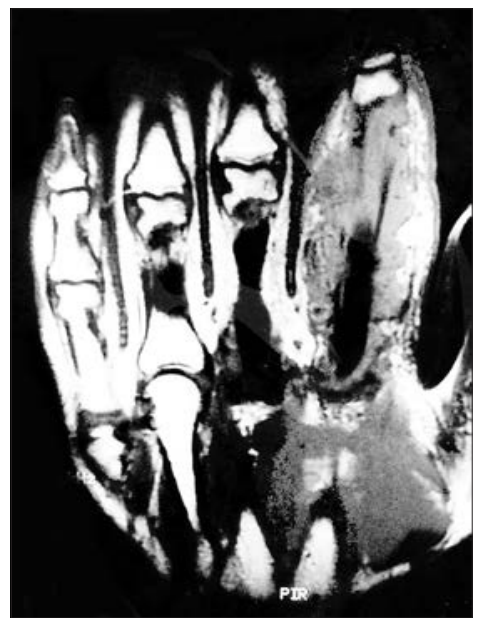

Figure 7: Magnetic resonance imaging of index finger with synovial hypertrophy 


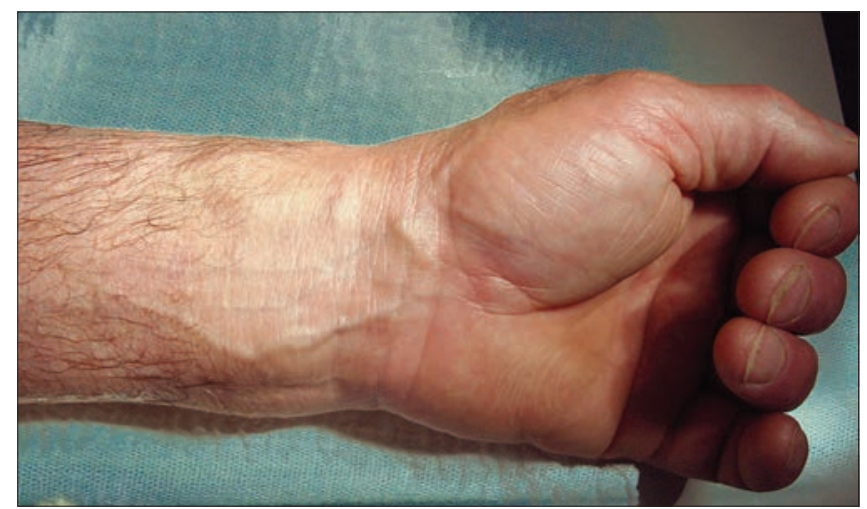

Figure 8: Volar wrist tumour

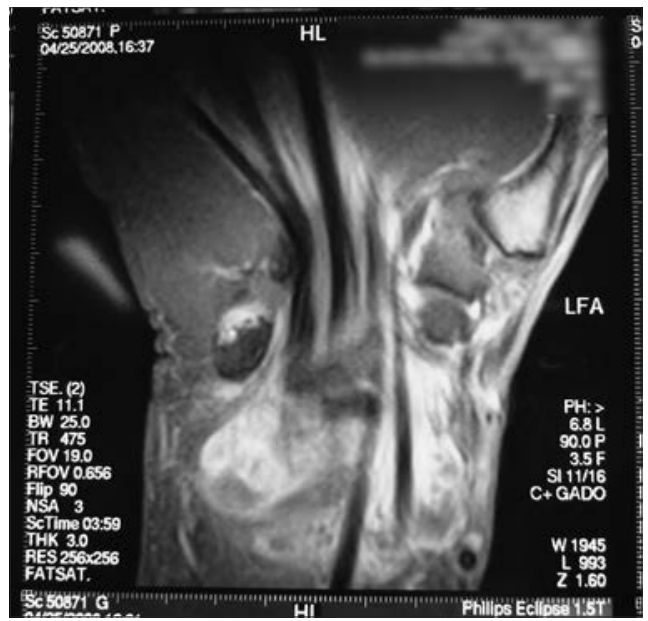

Figure 10: Computed tomography with peritendinous hypertrophy

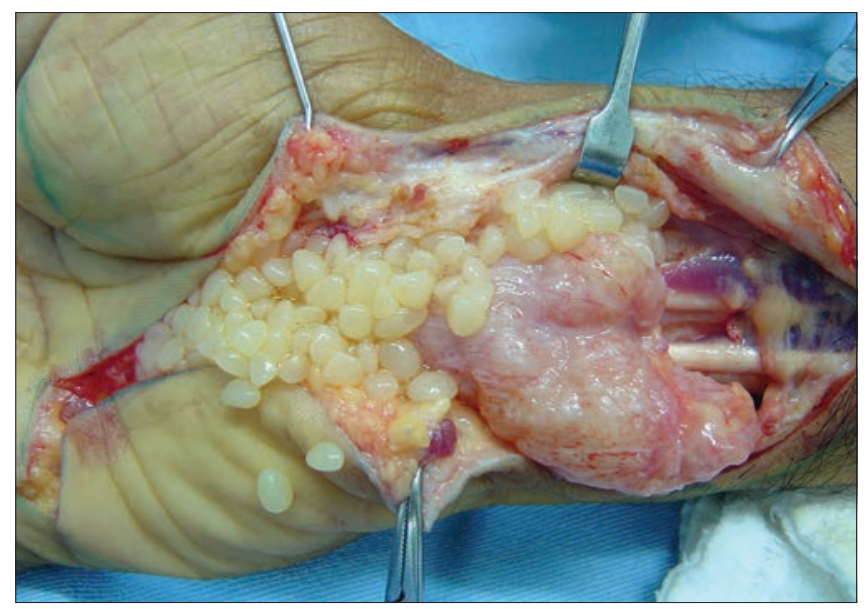

Figure 12: Extensive synovial hypertrophy and rice bodies

Case 3 result at 1-year post-operative after synovectomy and antituberculosis drugs treatment [Figure 14].

Case 5 result at 1-year after surgery and chemotherapy with antituberculosis drugs. Note the decrease in the volume of the thumb and fifth finger after conservative treatment in these specific areas [Figure 15].

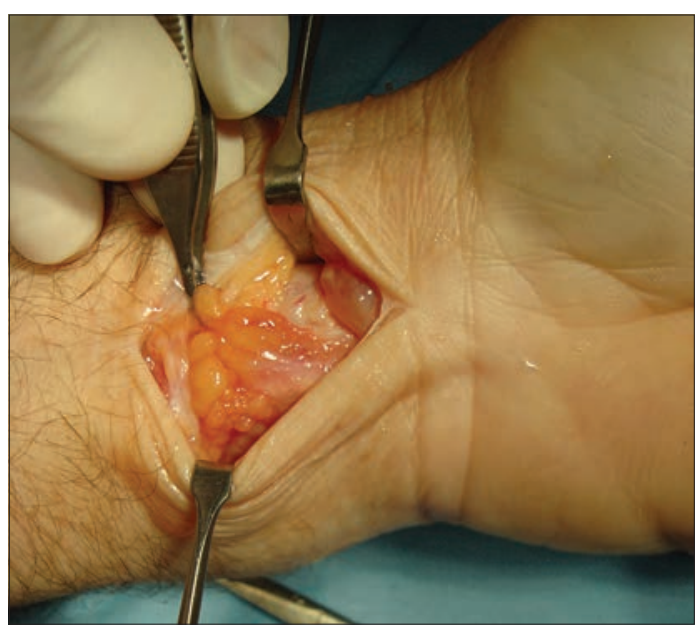

Figure 9: Rice bodies

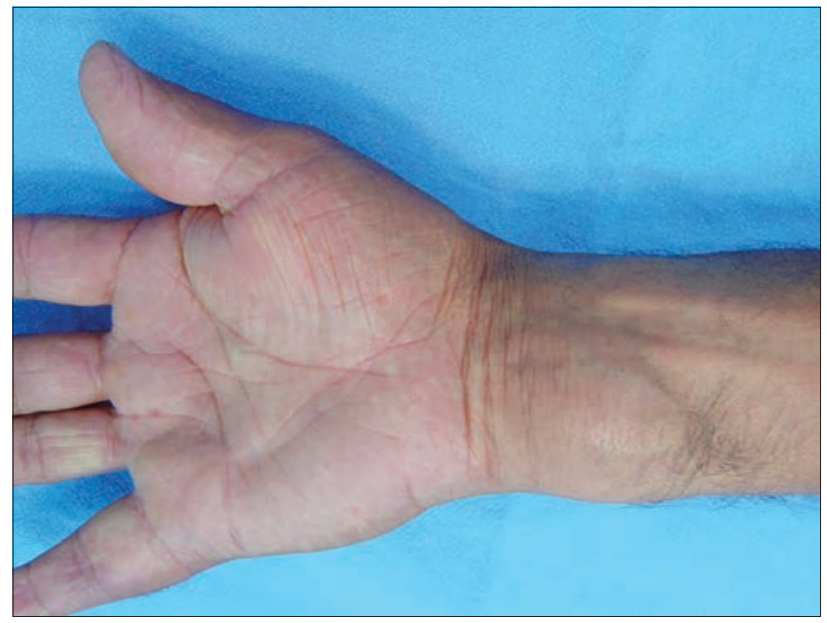

Figure 11: Wrist tumour

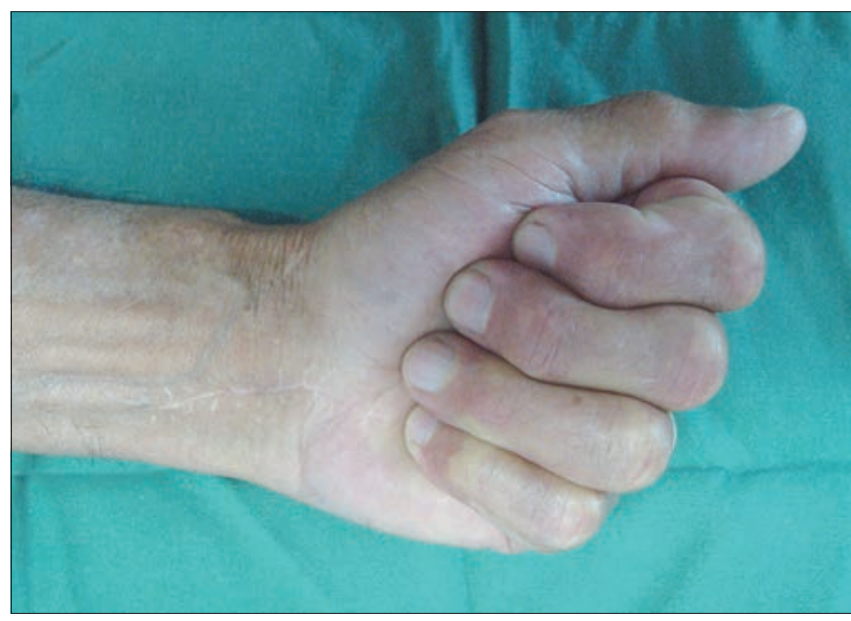

Figure 13: Functional result

\section{DISCUSSION}

Tuberculosis remains one of the most prevalent infectious diseases in the world. O-TBC accounts for $1 \%-3 \%$ of cases Indian Journal of Plastic Surgery Volume 50 Issue 2 May-August 2017 


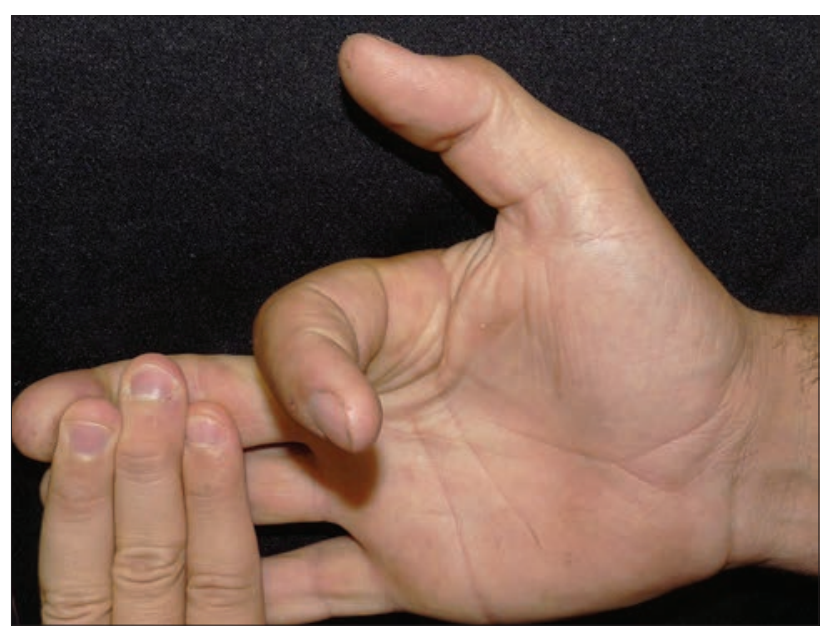

Figure 14: Adequate flexor movement

and $5 \%-10 \%$ of EP-TBC after pleural and lymph node disease. The commonly affected extrapulmonary sites are lymph nodes genitourinary tract, bone marrow, CNS and musculoskeletal. The pathogenesis may be direct inoculation or haematogenous spread from a primary focus. ${ }^{[6]}$

Arthritis caused by O-TBC presents as monoarticular and is located in the joints that support the body weight and are more vascularised. Hand tuberculosis presents as osteomyelitis associated with pain, swelling and functional limitation, with or without constitutional symptoms. Tenosynovitis though rare and is the most common presentation. The flexor apparatus is most affected, as well as the ulnar side when compared to the radial one; ; $^{[1-20]}$ tenosynovitis is more common in upper limb as compare to lower limb and commonly involves the flexor or volar site of wrist and hand of dominant limb; ;24] this detail was observed in the cases presented in a 5-year period in our hand surgery service. Tendon rupture is a common complication that was not observed in the cases discussed. Clinical presentation is like a slow-growing tumour, associated with local pain that worsens with movement of the fingers. The growth within the carpal tunnel produces median nerve compression. This was observed in one case. There are three histopathological stages of disease depending on duration of disease, resistance of patient and virulence of infecting agent; in early stage, there is vascular granulation tissue formation followed by obliteration of tendon sheath by fibrous tissue fluid accumulation and formation of rice bodies or sago seeds (which are fibrinous masses or tubercle made by caseation). In the final stage, there is rupture of tendon sheath, extensive caseation and granulation take place. ${ }^{[27]}$ The rice bodies are also commonly found in many diseases such as SLE, rheumatoid arthritis, seronegative arthritis and osteoarthritis of joint. ${ }^{[29]}$

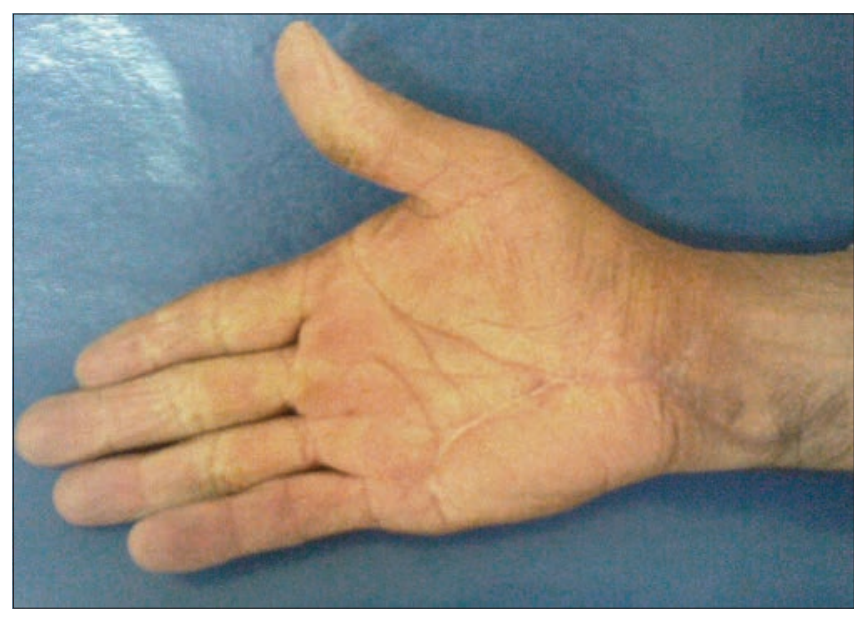

Figure 15: Final aesthetic result

Synovial biopsy is important for diagnosis and was performed in all patients (excisional biopsy) confirming the diagnosis by specific Ziehl-Neelsen staining and culture for AARB.

Treatmentshouldincludean antituberculosischemotherapy regimen of 12 months, as the WHO recommendations. The ideal time for a surgical approach is still controversial. In this case, synovectomy with surgical debridement of the hypertrophied tissue, with or without surgical repair of the flexor-extensor apparatus, can be performed.

The main treatment in all cases presented was the initial surgical approach. Management was removal of the hypertrophied synovial tissue and debridement of the affected area, combined with post-operative anti-tuberculosis chemotherapy for 1 year. To make a provisional diagnosis of tuberculosis and to start antituberculosis treatment, it is always better to rule out other common causes and conclude clinical, radiological, histopathological and PCR findings suggestive of tubercular tenosynovitis.

\section{CONCLUSIONS}

Tuberculosis remains one of the most prevalent infectious diseases worldwide. Osteoarticular involvement is infrequent and synovial infection in the hand is rare. When the disease is not treated in time and properly, consequences in the hand can be permanent in the flexor-extensor apparatus. Knowledge of this type of disease in the hand can provide a better diagnosis and outcome.

\section{Declaration of patient consent}

The authors certify that they have obtained all appropriate patient consent forms. In the form the patient(s) has/have 
given his/her/their consent for his/her/their images and other clinical information to be reported in the journal. The patients understand that their names and initials will not be published and due efforts will be made to conceal their identity, but anonymity cannot be guaranteed.

\section{Acknowledgement}

We thank Dr. Everton Braga Silva for his support during the anaesthetic procedures in all patients.

\section{Financial support and sponsorship}

Nil.

\section{Conflicts of interest}

There are no conflicts of interest.

\section{REFERENCES}

1. Malaviya AN, Kotwal PP. Arthritis associated with tuberculosis. Best Pract Res Clin Rheumatol 2003;17:319-43.

2. Fortún J, Martín-Dávila $P$, Rodríguez JM, Navas E, Moreno S. Mycobacterium tuberculosis infection. Medicine 2010;10:3808-19. DOI: 10.1016/S0304-5412(10)70119-0.

3. Jagtap SA, Sonawane DV, Saraogi AA. Isolated tuberculosis of scapula in a young adult. Int J Mycobacteriol 2013;2:114-7.

4. Pigrau-Serrallach $\mathrm{C}$, Rodríguez-Pardo D. Bone and joint tuberculosis. Eur Spine J 2013;22 Suppl 4:556-66.

5. Solsona G, Moltob Anna, Mateo L, Olive A. Keys for the diagnostic of tuberculosis arthritis. FMC 2009;16:272-5. DOI: 10.1016/S1134-2072(09)71272-1.

6. Higuchi $\mathrm{S}$, Ishihara $\mathrm{S}$, Kobayashi $\mathrm{H}$, Arai $\mathrm{T}$. A mass lesion of the wrist: A rare manifestation of tuberculosis. Intern Med 2008;47:313-6.

7. Carreño L, López F. Septic Arthritis Medicine 2001;8:1819-25.

8. lagnocco A, Coari G, Buzzi G, Guerrisi R, Valesini G. Magnetic resonance imaging of peripheral osteoarticular tuberculosis compared with sonography and standard radiographs. Rheumatol Int 2003;23:195-7.

9. Dlimi F, Abouzahir M, Mahfoud M, Berrada MS, El Bardouni A, El Yaacoubi M, et al. Multifocal bone tuberculosis: A case report. Foot Ankle Surg 2011;17:e47-50.

10. Pelayo G, Tinoco I, Sancha M, Martin A. Diagnostic and therapeutic protocol for the acute septic arthritis. Medicine 2010;10:3597-600. Available from: https://xa.yimg.com/kq/ groups/./artritis+aguda+septica.pdf. [Last accessed on 11 Oct 2017].

11. Ostrowska M, Gietka J, Nesteruk T, Piliszek A, Walecki J. Shoulder joint tuberculosis. Pol J Radiol 2012;77:55-9.

12. Mateo L, Ruiz J, Olivé A, Mari J, Perez R, Tena X, Prats M. Osteoarticular Tuberculosis: Study of 53 cases Med Clin
2007;129:506-9. DOI: 10.1157/13111371.

13. Martínez E, Mateos F, Blanch J, Botran S, Garcia Sanchez M. Extrapulmonary tuberculosis. Clinical types. Medicine 2010;10:3820-9. Available from: https://es.scribd.com/ document/312178838/Tuberculosis-Extrapulmonar-las-variasFormas-Clinicas. [Last accessed on 11 Oct 2017].

14. Mariconda M, Cozzolino A, Attingenti P, Cozzolino F, Milano C. Osteoarticular tuberculosis in a developed country. J Infect 2007;54:375-80.

15. Arathi N, Ahmad F, Huda N. Osteoarticular tuberculosis - A three years' retrospective study. J Clin Diagn Res 2013;7:2189-92.

16. Forse $\mathrm{CL}$, Mucha $\mathrm{BL}$, Santos ML, Ongcapin EH. Rice body formation without rheumatic disease or tuberculosis infection: A case report and literature review. Clin Rheumatol 2012;31:1753-6.

17. Dhillon MS, Singh P, Sharma R, Gill SS, Nagi ON. Tuberculous osteomyelitis of the cuboid: A report of four cases. J Foot Ankle Surg 2000;39:329-35.

18. HassanpourSE, Gousheh J. Mycobacterium tuberculosis-induced carpal tunnel syndrome: Management and follow-up evaluation. J Hand Surg Am 2006;31:575-9.

19. Seung OP, Sulaiman W. Osteoarticular tuberculosis mimicking rheumatoid arthritis. Mod Rheumatol 2012;22:931-3.

20. Aboudola S, Sienko A, Carey RB, Johnson S. Tuberculous tenosynovitis. Hum Pathol 2004;35:1044-6.

21. Kwan CK, Ernst JD. HIV and tuberculosis: A deadly human syndemic. Clin Microbiol Rev 2011;24:351-76.

22. Al-Qattan MM, Al-Namla A, Al-Thunayan A, Al-Omawi M. Tuberculosis of the hand. J Hand Surg Am 2011;36:1413-21.

23. Samuel $S$, Boopalan $P R$, Alexander M, Ismavel R, Varghese VD, Mathai $\mathrm{T}$, et al. Tuberculosis of and around the ankle. J Foot Ankle Surg 2011;50:466-72.

24. Pei-Hung S, Cheng-Mien C, Gua-Shu H, Shing-Sheng wu, ChianHer Lee. Tuberculous tenosynovitis of the flexor tendons of the wrist and hand. J Med Sci 2002;22:227-30. Available from: https:// www.jscimedcentral.com/Orthopedics/orthopedics-2-1039.pdf. [Last accessed on11 Oct 2017].

25. Rashid M, Sarwar SU, Haq EU, Islam MZ, Rizvi TA, Ahmad M, et al. Tuberculous tenosynovitis: A cause of carpal tunnel syndrome. J Pak Med Assoc 2006;56:116-8.

26. Marquet A, Moya L, Aldanondo I, Ernesto Muñoz-Zato, Rosario Carrillo, Pedro Jaén. Coexistence of verrucosa cutis tuberculosis and articular tuberculosis in the same patient. Actas Dermosifiliogr 2006;97:56-8. Available from: http:// www.actasdermo.org/es/coexistencia-tuberculosis-verrucosaarticular-un/articulo/13084926/. [Last accessed 11 Oct 2017].

27. Sharma SK, Ryan H, Khaparde S, Sachdeva KS, Singh AD, MohanA, etal. Index-TB guidelines: Guidelines on extrapulmonary tuberculosis for India. Indian J Med Res 2017;145:448-63.

28. Lall H, Nag SK, Jain VK, Khare R, Mittal D. Tuberculous extensor tenosynovitis of the wrist with extensor pollicis longus rupture: A case report. J Med Case Rep 2009;3:142.

29. Tyllianakis M, Kasimatis G, Athanaselis S, Melachrinou M. Rice-body formation and tenosynovitis of the wrist: A case report. J Orthop Surg (Hong Kong) 2006;14:208-11. 